\title{
Image Feature Extraction based on Pulse Coupled Neural Networks for Seafloor Sediment Classification
}

\author{
Chenchen Liu ${ }^{1}$ Zhimeng Zhang ${ }^{2}$ \\ ${ }^{1}$ Department of Computer and Information engineering, Shijiazhuang Railway Institution, Shijiazhuang 050043, \\ P. R. China \\ ${ }^{2}$ Department of Underwater Acoustic engineering, Harbin Engineering University, Harbin 150001, P. R. China
}

\begin{abstract}
For the reason of different images with different space distribution of gray levels, we proposed a texture representation based on simplified pulse coupled neural networks (PCNN) model which output a series of binary images corresponding to different gray levels. Then we transformed the images into 1D temporal sequence by calculating their variances to form feature vectors. Experiments show that the texture representation was rotation invariant which provided high classification rate for natural texture images. When used to classify side-scan sonar seafloor images of 12 types of sediment, accurate recognition rate of $100 \%$ was obtained. With the inherent parallel capability of PCNN, the method is more suited for real-time processing of sonar systems.
\end{abstract}

Keywords: Pulse coupled neural networks (PCNN), Feature extraction, Seafloor sediment classification, Side-scan sonar images

\section{Introduction}

Seafloor sediment research by means of acoustic echo is important for ocean engineering. The high resolution side-scan sonar images contain geomorphic and textual information which can be used for sediment classification or recognition [1].

The gray level of side-scan sonar image is correspondent to acoustic echo intensity which is a complicated physical parameter and associated with multi-factors such as the sonar transmit power, sediment type or incidence angle of acoustic waves and so on. Different sediment could have the same echo intensity [2], so it is impractical to judge the sediment type by the gray levels directly. In research, we found that the images of different sediments have different texture which could be used as a feature for sediment classification.

PCNN is a novel net model which imitates animal's retina. It can be unsupervised auto-learning and fit for real-time image processing. PCNN is a 2D neural network, references [4]-[6] used several methods of dimensionality reduction to transform PCNN output binary image sequence to 1D signal as the feature vectors for classification by which got perfect performance.

In section 2, we introduced a simplified PCNN model and a novel image texture extraction algorithm. Due to the difference in the distribution of the same grey-level value in images, the variance sequence of PCNN output binary images was used as textural representations of original images. In section 3, Experiment was done on both side-scan sonar images of seafloor sediment and natural texture images. And the results show that the proposed algorithm can get high classification rate.

\section{Feature extraction algorithm}

\subsection{PCNN model}

There is great dissimilarity of PCNN and traditional neural networks in structure and operating principle. Each of the PCNN neurons has a unique quantitative relation with one image pixel and mutual interaction with other peripheral neurons by link matrix. Furthermore, the firing threshold of neuron is time varying. Both of the characteristics make adjacent neurons in different intensity around the firing one to fire at the same time, so it is called synchronous pulsefiring [3] and can be used in image processing to get better effects. But according to the large non-linear factors and integrations of drain capacitance, the original PCNN modal parameters are analyzed and determined hard mathematically which restricts the application of PCNN. So G. Kuntimad and H. S. Ranganath gave a simplified PCNN model for image processing, just showed in Fig.1 (at the next page), in which PCNN neuron's inputs are link $L_{i j}$ and feedback $F_{i j}$ which only accepts external stimulus, then to form internal active item $U_{i j}$ with $L_{i j}$. When $U_{i j}$ is greater than dynamic threshold $\theta_{i j}$, the PCNN neuron would putout sequential pulse series $Y_{i j}$. 


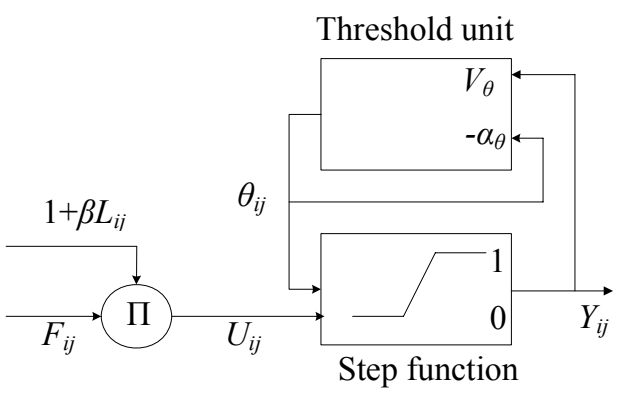

Fig. 1: Simplified PCNN model.

Based on the above simplified model, we give the PCNN iterative computing formulation, just as following function (1)-(5):

$$
\begin{gathered}
F_{i j}(n)=I_{i j} \\
L_{i j}(n)=\sum_{k l} w_{i j k l} Y(n-1) \\
U_{i j}(n)=F_{i j}(n) \\
\theta_{i j}(n)=\exp \left(-\alpha_{\theta}\right) \theta_{i j}(n-1)+V_{\theta} Y_{i j}(n-1) \\
Y_{i j}(n)= \begin{cases}1 & U(n) \geq \theta_{i j}(n-1) \\
0 & U(n)<\theta_{i j}(n)\end{cases}
\end{gathered}
$$

\subsection{Texture feature representation}

PCNN could putout a series of binary images which correspond to 2D distribution of different gray levels with suitable parameters when a grayscale image inputs it. The $1 \mathrm{D}$ temporal series can be derived from the binary images to form the invariable and unique feature representation for classification.

In this paper, we use the variances of PCNN outputting binary images as feature vectors to classify side-scan sonar images. Firstly, give the definition of temporal series,

$$
T(n)=V_{p}(n)
$$

where $V_{p}(n)$ is variance vector of output images at different time $n$. The variance is a statistic characteristic which exhibits difference between images. PCNN calculated the variance of each binary image in each iterative procedure at $n$ time and form a temporal series. The images with different texture would cause the distinct disparities in the number of firing neurons and correspondent firing time sequence because of the difference of gray level distribution. So the output images are varying with each input grayscale image and the correspondent variance-time series i.e. $T(n)$ is different from others.

\section{Experiments}

\subsection{On texture of sonar images}

The 12 side-scan sonar images of seafloor sediments which were used to verify the PCNN algorithm are just in followed Fig.2, the order of them is (1) to (12) from left to right and top down.
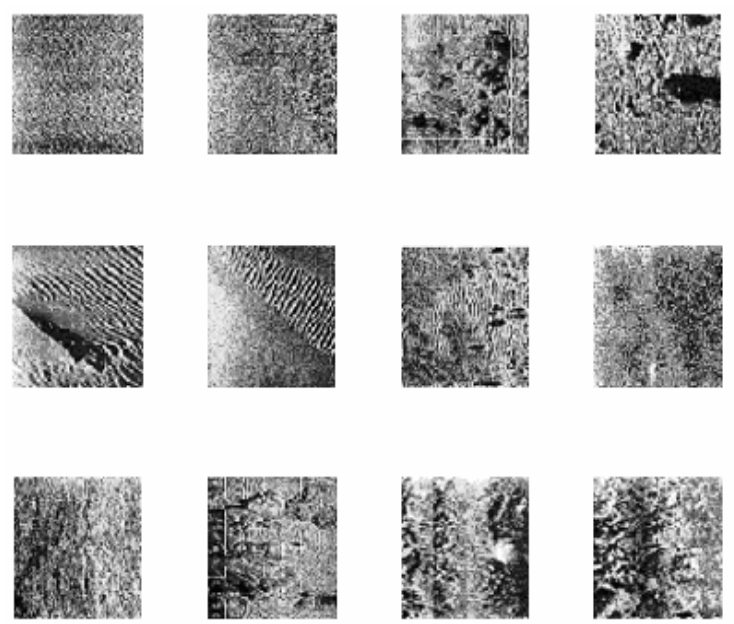

Fig. 2: Seafloor sediments images.

Experiment procedure is given as follows:

- Step 1. PCNN parameters setting: which is based on amounts of test experience, where attenuation coefficient $\alpha_{\theta}=0.05$, link coefficient $\beta=0.1$, internal link weighting matrix

$$
W=\left[\begin{array}{ccc}
0.44 & 1 & 0.44 \\
1 & 0 & 1 \\
0.44 & 1 & 0.44
\end{array}\right],
$$

and the greatest gray-level value was presupposed as the threshold $V_{\theta}$

- Step 2. Input the above 12 images into PCNN, and calculate the normal variance series of the output binary images. The variance series of image (1), (8), (9), (11) are showed in Fig.3 (a-d) at the next page, the difference of them could be seen distinctly.

- $\quad$ Step 3. Rotate each sonar image $30^{\circ}, 45^{\circ}$ and $90^{\circ}$ in Fig. 2 and obtain 48 sample images, then input them to PCNN and calculate interobject distance and cluster distance of obtained variance series (the feature vector) to show the feature performance. Results are just 
in following Table. 1 at the next column. In Table.1, the maximum mean inter-object distance is 0.2178 and the minimum cluster distance is 0.2522 , so the variance series can be used as effective feature for sonar image classification and can give the right answer to which type the seafloor sediments are.

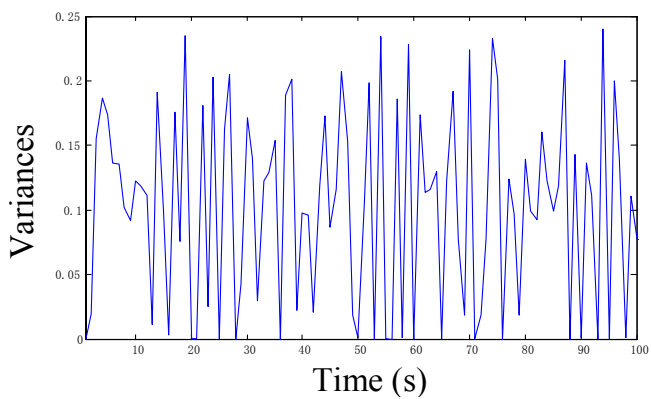

(a)

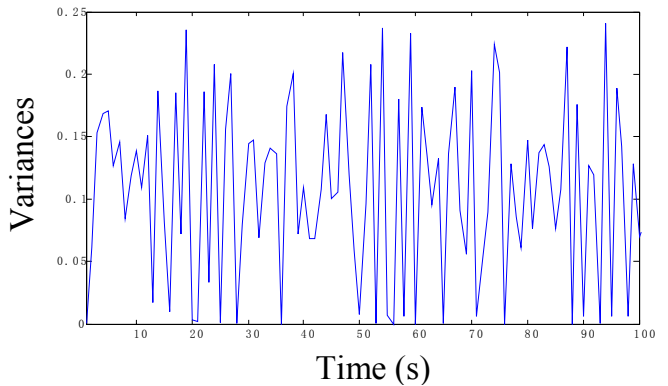

(b)

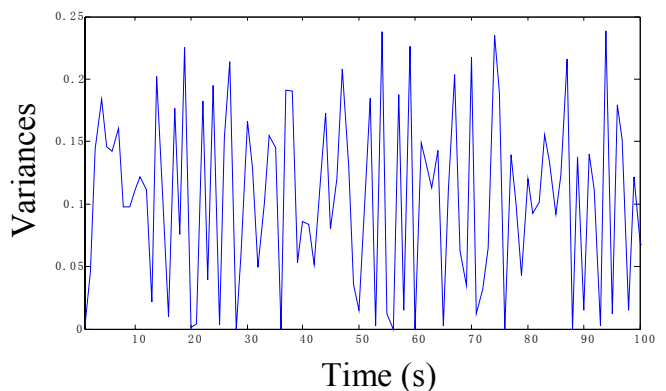

(c)

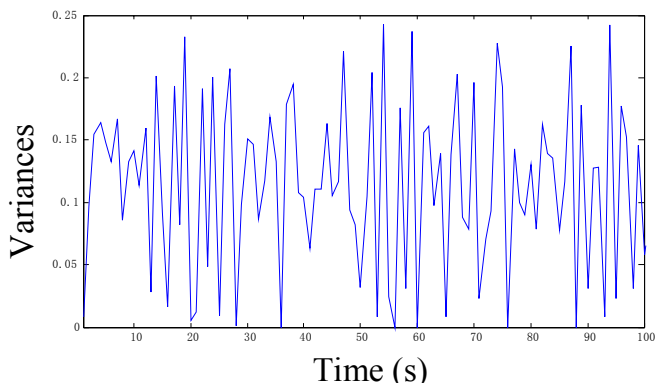

(d)

Fig. 3: Variance series of PCNN output images.

\begin{tabular}{|c|c|c|}
\hline Image & $\begin{array}{c}\text { Mean inter- } \\
\text { object distance }\end{array}$ & $\begin{array}{c}\text { Minimum } \\
\text { cluster distance }\end{array}$ \\
\hline 1 & 0.1103 & \multirow{5}{*}{0.2522} \\
\hline 2 & 0.1248 & \\
\hline 3 & 0.0458 & \\
\hline 4 & 0.0884 & \\
\hline 5 & 0.1555 & \\
\hline 6 & 0.0883 & $\begin{array}{c}\text { Maximum } \\
\text { cluster distance }\end{array}$ \\
\hline 7 & 0.1533 & \multirow{6}{*}{2.1061} \\
\hline 8 & 0.2178 & \\
\hline 9 & 0.0212 & \\
\hline 10 & 0.0709 & \\
\hline 11 & 0.0437 & \\
\hline 12 & 0.0813 & \\
\hline
\end{tabular}

Table 1: Performance of feature extraction by PCNN.

- $\quad$ Step 4. Match feature vectors of the 48 rotated images with those of 12 original images in advance, and use min-distance classifier for classification. Results show that the identification rate is $100 \%$. And it can also prove that the feature obtained by the proposed PCNN method is rotation invariant.

\subsection{On texture of natural images}

Because the number of side-scan sonar images is too small to reflect the classification performance accurately, we verified the proposed PCNN algorithm by 114 natural images in Brodatz texture database. There are some samples of the database in Fig.4.
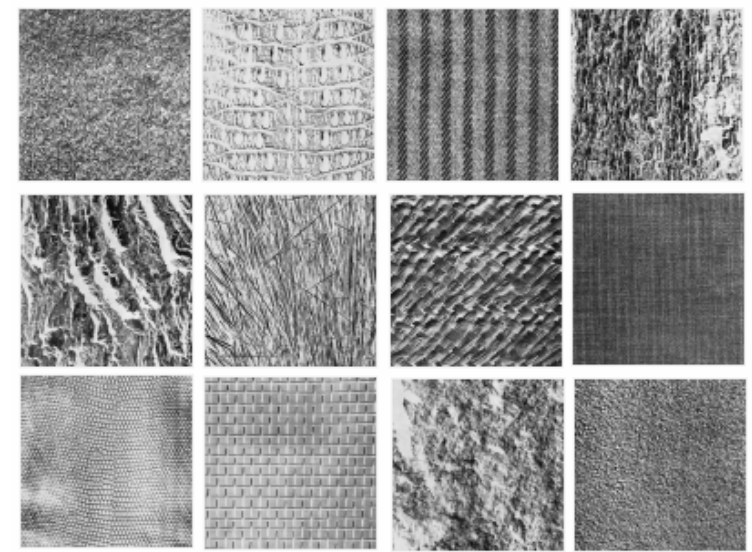

Fig. 4: Examples of Brodatz texture database.

Each of images was rotated $30^{\circ}, 45^{\circ}, 60^{\circ}$ and $90^{\circ}$ to compose 570 image samples and be divided into 270 for training and 300 for test. 
Then the PCNN method and other algorithms i.e. Fourier power spectrum, grayscale run-length matrix and neighborhood gray level co-occurrence were used to represent the texture feature of the sample images, then sent the feature vectors into BP neural networks classifier. The experiment results are showed in Table.2.

\begin{tabular}{|c|c|c|c|}
\hline Algorithm & $\begin{array}{c}\text { Recognitio } \\
\text { n rate of } \\
\text { training } \\
\text { samples } \\
(\%)\end{array}$ & $\begin{array}{c}\text { Recognitio } \\
\text { n rate of } \\
\text { test } \\
\text { samples } \\
(\%)\end{array}$ & $\begin{array}{c}\text { Recognitio } \\
\text { n rate of } \\
\text { all samples } \\
(\%)\end{array}$ \\
\hline $\begin{array}{c}\text { Fourier power } \\
\text { spectrum }\end{array}$ & 65.2 & 72.3 & 68.9 \\
\hline $\begin{array}{c}\text { Grayscale } \\
\text { travel-distance } \\
\text { matrix }\end{array}$ & 63.3 & 58.0 & 60.5 \\
\hline $\begin{array}{c}\text { Neighborhood } \\
\text { gray level co- } \\
\text { occurrence }\end{array}$ & 96.7 & 91.1 & 94.0 \\
\hline PCNN method & 98.7 & 97.0 & 97.8 \\
\hline
\end{tabular}

Table 1: Results of natural texture images.

From Table.2, we can see perfect classification performance of the proposed PCNN method in the paper. PCNN model also can be used in region or edge extraction of images and its internal parallelism is suited to real-time image processing.

\section{Conclusions}

In the paper, a novel image texture representation was proposed based on simplified PCNN model which obtained a series of images corresponding to different gray levels of original side-scan sonar or natural texture images. Then the output binary image sequence was transformed into $1 \mathrm{D}$ variance series to form feature vector by calculating variance of each image. Experiment results show that the obtained features are rotation invariant and the PCNN algorithm is a better method to judge the sediment types according to the high classification rate for both sonar and natural images. The simplified PCNN model used here is convenient to be realized by parallel hardware for real-time processing.

\section{References}

[1] C.Y. Yang, F. Xu and J. J. Wei, Sea-floor sediment classification using a neighbor-hood gray level co-occurrence matrix, Journal of Harbin Engineering University, 26: 561-564, 2005.

[2] S. Stanic, R. Goodman and K.B. R.Briggs, Shallow water bottom reverberation meaturements,
IEEE Journal of Oceanic Engineering, 23:203210, 1998.

[3] J.Y. Zhang, B.Y. Li, Feature Extraction on Image Smothness based on PCNNs, Computer simulation, 20: 103-105, 2003.

[4] J.L. Johnson, Pulse-coupled Neural Nets: Translation, Rotation, Scale and Intensity Signal Invariances for Images, Applied Optics, 33: 62396253, 1994.

[5] J. Waldemark, V. Becanovic and T. Lindbland, Hybrid Neural Networks for Automatic Target Recognition, IEEE International Conference on Systems,Man ,and Cybernetics, 10: 12-15, 1997.

[6] Y.D. Ma, L. Li and Y.F. Wang, Principle and application of Pulse coupled neural net-works, Science Press, Beijing, 2006. 